Tohoku J. Exp. Med., 2009, 218, 309-316

\title{
Lamin A/C Gene Mutations in Familial Cardiomyopathy with Advanced Atrioventricular Block and Arrhythmia
}

\author{
Akiko Saga, ${ }^{1}$ Akihiko Karibe, ${ }^{1}$ Jun Otomo, ${ }^{2}$ Kaoru Iwabuchi, ${ }^{3}$ Toshiaki Takahashi, ${ }^{4}$ \\ Hiroyuki Kanno, ${ }^{5}$ Junichi Kikuchi, ${ }^{6}$ Mitsumasa Keitoku, ${ }^{2}$ Tsuyoshi Shinozaki ${ }^{7}$ and \\ Hiroaki Shimokawa ${ }^{1}$
}

\author{
${ }^{1}$ Department of Cardiovascular Medicine, Tohoku University Graduate School of Medicine, Sendai, Japan \\ ${ }^{2}$ Department of Cardiology, Tohoku Kosai Hospital, Sendai, Japan \\ ${ }^{3}$ Department of Cardiology, Osaki City Hospital, Osaki, Japan \\ ${ }^{4}$ Second Department of Internal Medicine, Hiraka General Hospital, Yokote, Japan \\ ${ }^{5}$ Department of Cardiology, Katta General Hospital, Shiroishi, Japan \\ ${ }^{6}$ Second Department of Internal Medicine, Towada Municipal Central Hospital, Towada, Japan \\ ${ }^{7}$ Department of Cardiology, Sendai Medical Center, Sendai, Japan
}

\begin{abstract}
Lamin $\mathrm{A}$ and $\mathrm{C}$ proteins, encoded by the lamin $\mathrm{A} / \mathrm{C}$ gene $(L M N A)$, are inner nuclear membrane proteins predominantly expressed in terminally differentiated cells. Mutations in LMNA can cause various forms of cardiomyopathy with arrhythmia in an autosomal dominant manner. We collected and evaluated the clinical characteristics of unclassified familial cardiomyopathy with advanced AV block and sporadic cases with advanced AV block. Mutation in $\angle M N A$ was directly screened using the cycle sequencing method in 5 probands of the familial cardiomyopathy and 60 sporadic cases with advanced AV block. In four of the five familial cases $(80 \%)$, we identified four distinct mutations: two protein-truncation mutations, R225X and 815_818delinsCCAGAC, and two missense mutations, Y259H and R166P. No sporadic cases carried $\angle M N A$ mutation. Left ventricular end-diastolic diameter (LVEDD) was slightly enlarged in LMNA mutant carriers $(123.5 \pm 9.5 \%)$ as well as in non-carriers $(125.1 \pm 13.3 \%)$, while left ventricular fractional shortening (LVFS) was preserved in $\angle M N A$ mutant carriers $(32.3 \pm 4.8 \%)$ and non-carriers $(37.6 \pm 6.8 \%)$. In $L M N A$ mutation carriers, the average age at onset of advanced AV block is significantly lower than that in non-carriers ( $43.7 \pm 9.5$ vs. $65.3 \pm 13$ yr., $p<0.01)$. Ventricular tachycardia, sudden death, and poor prognosis were observed in $\angle M N A$ mutation carriers. $L M N A$ mutation could cause familial cardiomyopathy with insignificant LV remodeling, early-age onset of advanced AV block, and lethal ventricular arrhythmia. Screening of $L M N A$ mutation might be beneficial for risk stratification and clinical management of this type of unclassified familial cardiomyopathy. Cardiomyopathy; atrioventricular block; ventricular tachycardia; sudden death; molecular genetics.
\end{abstract}

Tohoku J. Exp. Med., 2009, 218 (4), 309-316. 두 2009 Tohoku University Medical Press

Cardiomyopathy is a primary myocardial disease that can cause sudden cardiac death and heart failure. Historically, cardiomyopathy has been recognized and classified with ventricular tissue remodeling, such as dilated, hypertrophic, and restrictive, as seen in 1996 World Health Organization (WHO)/International Society and Federation of Cardiology (ISFC) Task Force classification (WHO/ISFC Task Force. 1996). Arrhythmia is another important clinical feature of cardiomyopathy (Maron et al. 2006). Cardiac conduction disturbance and/or lethal ventricular arrhythmia are the major recognized phenotypes of arrhythmia. Progressive cardiac conduction disturbance is frequently observed in various types of myocardial diseases and affects the disease prognosis (Benson 2004). Hasegawa et al.
(1990) reported high prevalence of a non-hypertrophic and non-dilated type of cardiomyopathy with cardiac conduction disturbance and/or arrhythmia in their cardiac biopsy specimens. To date, the clinical features and etiology of this disease type has not been clarified.

Lamin $\mathrm{A}$ and $\mathrm{C}$ proteins are inner nuclear membrane proteins (Stuurman et al. 1998) and are predominantly expressed in terminally differentiated cells, including cardiomyocytes (Rober et al. 1989; Broers et al. 1997). The lamin $\mathrm{A} / \mathrm{C}$ gene (LMNA), located in the human chromosome 1q21.2, encodes two main isoforms of lamin $\mathrm{A}$ and $\mathrm{C}$ proteins by alternative splicing (Lin and Worman 1993). Heterozygous mutation in LMNA can cause dilated cardiomyopathy (DCM) with AV conduction disturbance (Kass et

Received March 4, 2009; revision accepted for publication June 25, 2009. doi:10.1620/tjem.218.309

Correspondence: Akihiko Karibe, Department of Cardiovascular Medicine, Tohoku University Graduate School of Medicine, 1-1

Seiryo-machi, Aoba-ku, Sendai 980-8574, Japan.

e-mail: akaribe@mail.tains.tohoku.ac.jp 
al. 1994; Fatkin et al. 1999) in an autosomal dominant manner. Cardiomyopathy associated with LMNA mutation is characterized by a variety of phenotypes, including supraventricular arrhythmia, ventricular arrhythmia, and skeletal muscle involvement, rather than the pure form of DCM (Brodsky et al. 2000; Taylor et al. 2003). For LMNA mutation in DCM patients, a prevalence as high as $33 \%$ has been reported; this shows that the gene considerably contributes to the development of cardiomyopathy (Arbustini et al. 2002).

The purpose of this study was to examine whether LMNA mutation is involved in some types of cardiomyopathy with cardiac conduction disturbance and/or arrhythmia and to evaluate the specific clinical characteristics of the disease.

\section{Methods}

\section{Patient enrollment}

We sequentially enrolled cases of advanced atrioventricular (AV) block from the Tohoku University Hospital and its five affiliated hospitals, from April 2003 to August 2006. Advanced AV block was diagnosed on the basis of progressive conduction disturbance, such as high-degree AV block and/or chronic atrial fibrillation with low ventricular rhythm $(<40 \mathrm{bpm})$ on 12-lead ECG and/or and 24-hour ambulatory ECG monitoring. We identified 5 cases of familial cardiomyopathy (see Fig. 1) and 60 sporadic cases (see Table 2) with advanced AV block. Detailed clinical information was obtained for each subject, including family history, age of manifestation, initial symptoms, New York Heart Association (NYHA) heart failure classification, physical examination findings, echocardiogram (2D, M-mode, and color Doppler), and measurement of serum creatine kinase (MM isoform) levels. Left ventricular remodeling was assessed with left ventricular end-diastolic diameter (LVEDD): the percentage of predicted value corrected for age and body surface area. And left ventricular systolic function was assessed with left ventricular fractional shortening (LVFS): the percentage of change in left ventricular dimension with systole.

Family members showing AV block and sudden cardiac death were designated as affected. Familial cardiomyopathy was recognized if at least two family members were affected (Mestroni et al. 1999). Phenotypic characterization was updated with additional clinical information and was expanded to include information on family members. All cases with advanced AV block secondary to ischemic heart disease, valvular heart disease, myocarditis, skeletal myopathy, autoimmune disease, surgical complication, and other apparent causes were excluded. This study was approved by the Ethics Committee of Tohoku University School of Medicine. All participants of this study gave informed consents for the clinical and genetic analysis.

\section{Mutation screening of LMNA}

Mutation screening of LMNA was performed according to the procedures described in the literature (Otomo et al. 2005). We obtained 10-15 mL of peripheral venous blood samples from each participant. Genomic deoxyribonucleic acid (DNA) was extracted from white blood cells with the QIAquick DNA blood kit (QIAGEN, Santa Clarita, California). Twelve coding exons of LMNA were amplified by polymerase chain reaction (PCR) with primers derived from intronic sequences. We purified $20 \mu \mathrm{L}$ of PCR products using the QIAquick PCR purification kit (QIAGEN). Bidirectional direct sequencing analysis was performed with an automated fluorescence sequencer, ABI PRISM 310 (Applied Biosystems, Foster City, California), by the dye-primer cycle sequencing method previously described (the BigDye Terminator v3.1 cycle sequencing kit, Applied Biosystems, and the DyeEx spin kit, QIAGEN) (Otomo et al. 2005). Total RNA was purified from white blood cells with the RNeasy mini kit (Qiagen) and subjected to reverse transcription as described in the literature (Otomo et al. 2005). A cDNA fragment containing the exonexon junction of interest was amplified by PCR. The PCR products were size-separated by $5 \%$ NuSieve GTG agarose (FMC BioProducts, Rockland) gel electrophoresis.

To confirm the direct sequencing data and to test whether the mutation was a polymorphism, we performed either the PCR-based restriction fragment-length polymorphism analysis or enzyme-mismatch cleavage analysis on both affected and non-affected family members, as well as 172 ethnically matched controls (344 chromosomes, randomly selected from our control genomic store, $>30$ years of age). For an analysis of predicted change in the amino acid sequence, the altered residues were examined for conservation across the species.

\section{Statistical analysis}

Quantitative results were expressed as means \pm standard deviation. Differences in continuous variables were analyzed using Student's $t$ test. Survival curves were estimated by the Kaplan-Meier method. A value of $p<0.05$ was considered statistically significant.

\section{Results}

Mutation screening of LMNA in familial cardiomyopathies and sporadic cases with advanced AV block

Mutation screening of all translated exons of LMNA was subsequently carried out on the 5 probands of familial cardiomyopathy (Table 1 and Fig. 1) and 60 sporadic cases with advanced AV block (Table 2) by PCR amplification of genomic DNA. In four of the five families, we identified four distinct mutations: a known one, R225X, and three novel mutations, 815_818delinsCCAGAC, Y259H, and R166P. No additional mutation was identified in the coding region of $L M N A$ in the four probands with defined mutation. We also detected four known synonymous single-nucleotide polymorphisms (SNPs), 1017G > A, 1338T $>$ C, 1551G $>$ A, and $1698 \mathrm{C}>\mathrm{T}$ in the $L M N A$ coding region of our 60 sporadic cases. We further identified a single base-pair change in intron 5 (IVS5-8C > G) in one case. This single basepair change was not detected in the controls. However, the base-pair change could not be considered a disease-related SNP because no mRNA splicing abnormality was found.

In the Family A proband III-2, we identified a novel LMNA mutation with deletion of an ACAA and insertion of a CCAGAC sequence at nucleotide number 815 to 818 , which is abbreviated as $815 \_818$ delinsCCAGAC (Fig. 2A). The mutation predicts a frameshift that is expected to completely alter the amino acid sequence that started from codon 272 and terminated at codon 480 in coil 2 of lamin A and $\mathrm{C}$ proteins (Fig. 2B). We then performed sequencing of exons harboring the $L M N A$ mutation for other family mem- 
Table 2. Clinical characteristics of patients with sporadic AV blocks.

\begin{tabular}{lc}
\hline Male, $\%(n=60)$ & 51 \\
Age of PMI, yrs $(n=60)$ & $62.6 \pm 15.1$ \\
Basal rhythm, \% $(n=60)$ & 94 \\
$\quad$ SR & 6 \\
AF/AFL & 64 \\
AVB degree, \% $(n=60)$ & 36 \\
$\quad$ II ${ }^{\circ}$ AVB & \\
III' AVB & 11 \\
AVB site, $n(n=23)$ & 12 \\
AHB & \\
HH'B, HVB & 95 \\
NYHA, class, \% $(n=60)$ & 5 \\
I & \\
II & \\
Echocardiography at PMI $(n=40)$ & $36.0 \pm 4.8$ \\
LAD, mm & $125 \pm 13.3$ \\
LVEDD, \% & $37.6 \pm 6.8$ \\
FS, \% &
\end{tabular}

Values are given as mean \pm S.D.

PMI, pacemaker implantation; SR, sinus rhythm; AF, atrial fibrillation; AFL, atrial flutter; AVB, atrioventricular block; AHB, atrio-His block; HH'B, intra-Hisian block; HVB, His-ventricular block; NYHA, New York Heart Association; LAD, left atrial diameter; LVEDD, left ventricular end-diastolic diameter; FS, fractional shortening.

bers to determine their mutation carrier status. We also carried out restriction-enzyme analysis using $M s c \mathrm{I}$ for the family for demonstrating the segregation of the mutation (Fig. 1). In Family B III-5, we identified a nonsense mutation, R225X (Fig. 2A). This mutation was confirmed by enzymemismatch cleavage analysis. The mutation predicts termination of amino acid coding at codon 225 and truncation in the coillb domain of lamin A and C proteins (Fig. 2B). This mutation was reported to be linked to DCM with conduction disturbance (Jakobs et al. 2001). In the Family C proband III-8, we identified a novel LMNA mutation, Y259H. The mutation was confirmed by enzyme-mismatch cleavage analysis. This mutation predicts substitution of the evolutionally highly conserved hydrophobic amino acid tyrosine (abbreviated as Y) for the basic amino acid histidine (abbreviated as H) at codon 259 in the coil 2 domain (Fig. 2A and 2C). In the Family D proband III-2, a novel LMNA mutation, R166P, was identified, and confirmed by restriction enzyme $M s p$ I analysis. The evolutionally highly conserved basic amino acid arginine (abbreviated as R) is substituted for the hydrophobic amino acid proline (abbreviated as $\mathrm{P}$ ) at codon 166 in the coil1b domain (Fig. 2A and 2C). No mutation was present in the 172 unrelated individuals. LMNA mutations accounted for $80 \%$ (4/5) of familial cardiomyopathy with advanced AV block. 


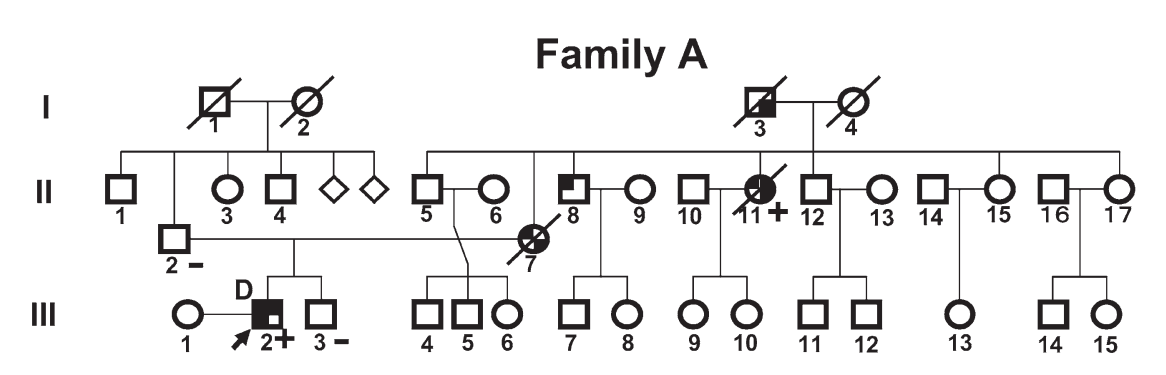

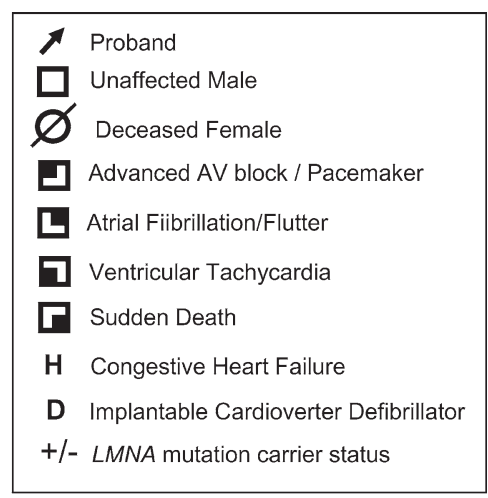
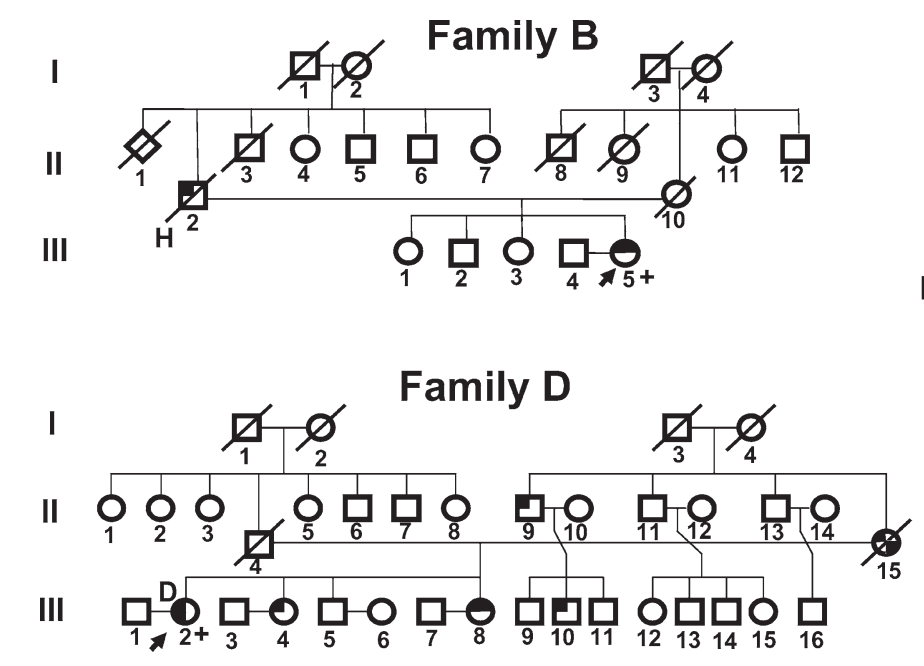
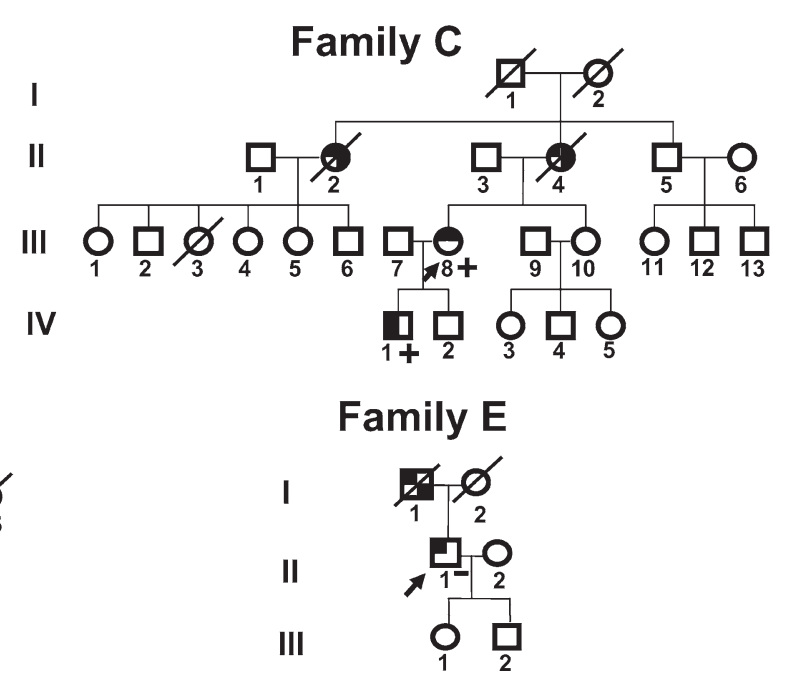

Fig. 1. Pedigree structure, and genotype and phenotypic features of the familial cardiomyopathy with advanced AV block. Cardiac phenotypes were variably expressed, designated by filled quadrants within pedigree symbols as indicated in the figure. All the affected members manifested advanced AV block.

\section{Clinical characteristics of LMNA mutation carriers and non-carriers with advanced AV block}

In 60 cases of sporadic advanced AV block, without LMNA mutation, the mean age of onset was $62.6 \pm 15.1$ years (Table 2). Most cases were in NYHA class I, but five patients were in NYHA class II. Echocardiogram showed slightly enlarged LVEDD (125.1 $\pm 13.3 \%)$ and preserved LVFS $(37.6 \pm 6.8 \%)$ at the onset of advanced AV block $(n=$ 40). Cardiac electrophysiological study (EPS) showed that advanced AV block occurred at the AV nodal level (AH block in EPS) in 11 patients and at the infra-His nodal level in 12 patients.

Detailed clinical data of the four probands and two affected members with the LMNA mutations were available (Table 1). These affected members had symptoms categorized as NYHA class I at onset. Advanced AV block was manifested at a mean age of $43.7 \pm 9.5$ years $(n=5)$, significantly lower than in sporadic cases $(p<0.01)$. EPS revealed that the site of $\mathrm{AV}$ block was at the $\mathrm{AH}$ level in Family A III-2 and Family C IV-1, and at the AH and HV levels in Family D III-2. Echocardiogram performed on the affected members showed slightly enlarged LVEDD (123.5 $\pm 9.5 \%, n=4)$ and preserved LVFS $(32.3 \pm 4.8 \%, n=4)$, with no significant difference between the familial cardiomyopathy and sporadic cases at onset. LVEDD (127.6 \pm $7.8 \%, n=3)$ and LVFS $(28.7 \pm 10.5 \%, n=3)$ were still preserved when the implantable cardioverter defibrillator (ICD) was inserted $(51.7 \pm 4.7$ years, $n=3)$ (Table 1$)$. There was no skeletal myopathy or increase in serum creatine phosphokinase levels.

Affected family members manifested a variety of arrhythmias besides advanced AV block (Fig. 1). Atrial fibrillation or atrial flutter was documented in Family A II-11 and III-2; Family B III-5; Family C II-2, II-4, and III-8; and Family D III-8. Ventricular tachycardia (VT) was documented in Family A III-2, Family C IV-1, and Family D III-2, and ICD therapy was performed on Family A III-2 and Family D III-2. Sudden death was observed in Family A I-3, II-7, and II-11; Family C II-2 and II-4; and Family D II-15. Affected family member II-11 of Family A died suddenly, even after pacemaker implantation. Prognosis of the LMNA mutation carriers in our cohort was poor, as previously reported for those with DCM (Taylor et al. 2003). The mean survival age for patients with $L M N A$ mutation $(n=12)$ was $58.3 \pm 8.6$ years. The cause of death included lethal arrhythmia $(n=7)$, thromboembolic stroke attributed 


\section{A. Identified LMNA mutations}

Family A III-2

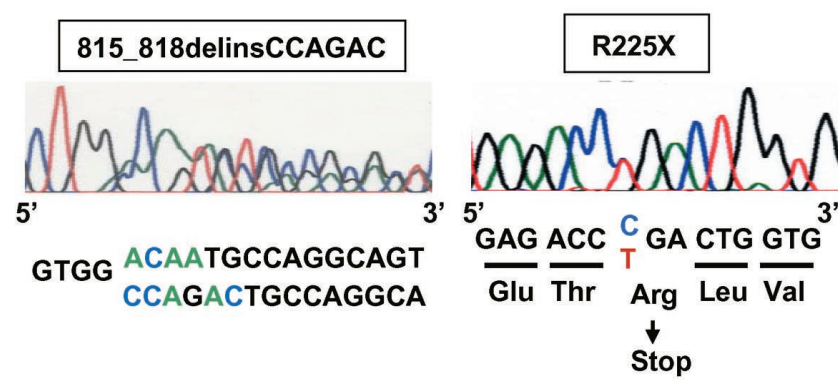

\section{B. Predicted truncation sites}

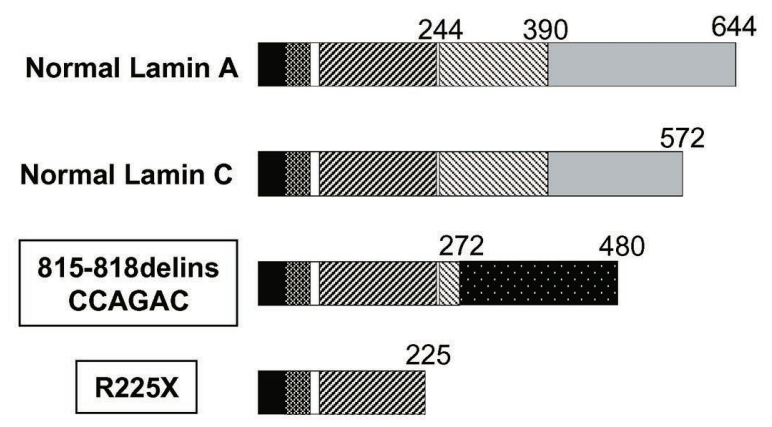

Family C III-8

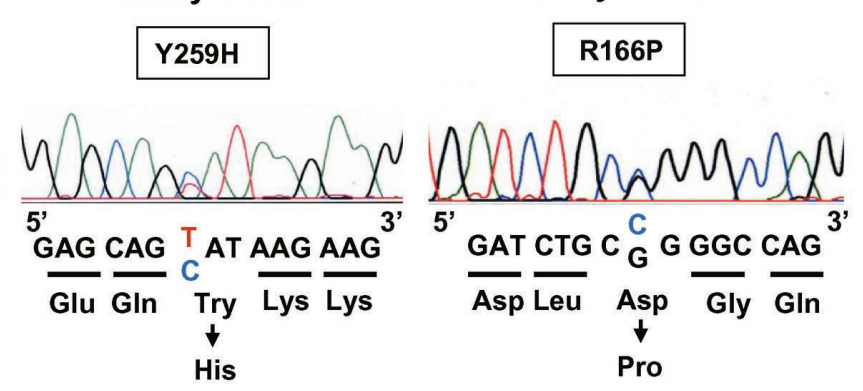

C. Homolog of the mutation sites
R166P

Fig. 2. LMNA mutations and predicted effects of the mutations in four families with advanced AV block. A. Mutation screening of LMNA was performed with the direct sequencing method. In the probands of the four families (Family A III-2, Family B III-5, Family C III-8, and Family D III-2), heterozygous variations in DNA sequence were detected as overlapped base-pair calls in contrast to single peak base-pair calls in wild type. B. The LMNA mutation 815 818delinsCCAGAC causes a frameshift at codon 272 and premature termination of translation at codon 480 . The $L M N A$ mutation R225X causes a protein truncation of lamin A and C proteins at codon 225. C. LMNA mutations Y259H and $\mathrm{R} 166 \mathrm{P}$ alter highly conserved amino acids among the species.

to atrial fibrillation $(n=4)$, and congestive heart failure $(n=$ 1).

\section{Discussion}

In this study, we identified one known and three novel LMNA mutations in a series of familial cardiomyopathy cases with advanced AV block and arrhythmia. We demonstrated that cardiomyopathy associated with LMNA mutation has unique characteristics, including early-age onset of AV nodal conduction block, complication of lethal ventricular arrhythmias, and poor prognosis.

\section{LMNA mutations and disease mechanisms}

Heterozygous mutation in LMNA can cause various types of familial diseases in striated muscle, peripheral nerve, and fat tissue, and systemic progeria syndrome. The disease caused by LMNA mutation is called "laminopathy" (Burke and Stewart 2002; Eriksson et al. 2003; Hennekam 2006). Heterozygous mutation, which causes amino acid substitution or decreased gene dosage (haploinsufficiency), can lead to an autosomal dominant familial disease with high penetrance. Four hypotheses have been proposed for the molecular mechanisms of LMNA mutation: (a) loss of structural integrity, (b) altered gene expression, (c) impaired progenitor cell proliferation, and (d) accumulation of toxic prelamin A (Broers et al. 2006). How LMNA mutations affect molecular and cellular mechanisms of each organ and cause the particular disease still remains to be elucidated.

Of the LMNA mutations identified in this study, two were from protein truncation, R225X and 815_818delinsCCAGAC, and two from amino acid substitution, Y259H and R166P. The two protein-truncation mutations are predicted to cause haploinsufficiency in normal lamin A and $\mathrm{C}$ proteins. The two amino acid-substitution mutations are very rare polarity-change substitutions of amino acids for evolutionally highly conserved amino acids and are predicted as a secondary-structure change within the rod domain of lamin $\mathrm{A}$ and $\mathrm{C}$. These four mutations are predicted to have the same disease causing effects as the previously reported LMNA mutations. Moreover, no recognizable differences between the truncation mutation and missense mutation cases were found with regard to severity of clinical symptoms.

Cardiomyopathy associated with LMNA mutation

In the LMNA mutation carriers, LVEDD was slightly 
enlarged but LVFS preserved, similar to sporadic advanced AV block without LMNA mutation. Cardiomyopathy associated with LMNA mutation was indistinguishable from other idiopathic forms of advanced AV block diagnosed with the usual clinical procedures.

It has been previously demonstrated (Taylor et al. 2003) that cardiac phenotypes of $L M N A$ mutations vary in DCM with and without AV block, and in mild forms of DCM. More variation may exist according to the cardiac phenotypes of LMNA mutation carriers. A meta-analysis of the clinical characteristics of 299 carriers of LMNA mutations demonstrated that the major cardiac phenotypes with LMNA mutations are cardiac dysrhythmias (92\%), including supraventricular arrhythmia, AV conduction disturbance, and VT after the age of 30 years, rather than heart failure $(64 \%)$ after the age of 50 years (van Berlo et al. 2005). In our patient cohort, the cardiac phenotype of LMNA mutation was advanced AV block with insignificant left ventricular (LV) remodeling and ventricular arrhythmia.

No LMNA mutation was found in a previous mutation screening on 100 Japanese DCM patients (Shimizu et al. 2005). Their patient enrollment criteria were as follows: echocardiographic demonstration of depressed systolic function of the LV (LV ejection fraction $<0.45$ and/or fractional shortening $<0.25$ ) and a dilated LV (LV end-diastolic dimension $>117 \%$ of the predicted value corrected for age and body surface area). Patient population of our study was different from their patient population because of the preserved LV systolic function. It is conceivable that some cases of LMNA mutations may be treated as unclassified familial atrial fibrillation, AV block, VT, or sudden death. These cardiac phenotypes resemble the "arrhythmia type of cardiomyopathy" previously proposed by Sekiguchi et al. (2008). LMNA mutation may contribute in part to this type of cardiomyopathy. Further studies are needed to confirm this hypothesis.

\section{Progressive cardiac conduction disease in LMNA muta- tion carriers}

Two probands with two different LMNA mutations showed an AH block and one proband showed AH and HV blocks. In our LMNA mutation carriers, advanced AV block seemed to occur in the intra-AV nodal conduction system and expanded to the infra-His bundle level. Furthermore, VT was manifested by the affected cases (Family A III-2, Family C IV-1, and Family D III-2) (Fig. 1). One of the probands showed left bundle branch reentrant monomorphic VT, which could be managed by radio-frequency catheter ablation therapy. Expanded degeneration of the HisPurkinje conduction system may contribute to the substrate for VT. We have previously reported that AV nodal and LV myocardial degeneration were associated with LMNA mutations in autopsy cases with DCM (Otomo et al. 2005). Wolf et al. (2008) reported that lamin A/C haploinsufficiency caused early-onset programmed cell death of AV nodal myocytes and progressive electrophysiological disease in their mouse model (Wolf et al. 2008). However, they could not reveal the underlying molecular cellular events that led to apoptosis in the cardiac conduction system in their model.

In general, AV block was caused by various types of acute and chronic myocardial injury, including myocardial infarction, myocarditis, skeletal myopathy, maternal antibodies, and surgical complications. However, the most frequent cause of AV block is fibrosis of unknown etiology (Waller et al. 1993). In this study, we could not find LMNA mutations in 60 sporadic cases with advanced AV block. The contribution of LMNA mutations in sporadic cases was presumed to be insignificant (less than 0.83\%). Age at onset of $\mathrm{AV}$ block in the sporadic cases was quite different from that in the familial cases, suggesting different underlying etiologies.

\section{Screening for LMNA mutations in patients with advanced AV block}

Patients implanted with a pacemaker due to highdegree AV block and/or atrial fibrillation had a worse prognosis than those with pacemaker due to sick sinus syndrome (Brunner et al. 2004). The incidence of second-degree AV block in DCM patients was 9.5\% (Schoeller et al. 1993). AV block complications were also recognized as poor prognostic factors (Xiao et al. 1996) and required specific clinical management. It should be noted that some AV block patients have a poor prognosis even after pacemaker implantation because of subsequent manifestation of other ventricular arrhythmia or heart failure. To date, no information has been gathered on risk stratification in patients with advanced AV block. Mutations in alpha-subunit of cardiac sodium channels gene ( $S C N 5 A$ ) have been associated with progressive AV block (Schott et al. 1999) and heart failure (Olson et al. 2005). SCN5A could be a genetic predisposing factor for "high-risk" advanced AV block. However, it has not yet been clarified.

In this study, we demonstrated that LMNA mutation carriers showed poor prognosis due to ventricular arrhythmia and sudden death, and pacemaker therapy alone may not be sufficient. Prophylactic implantation of ICD may be beneficial for prevention of sudden death in patients with LMNA mutation (Meune et al. 2006). Indeed, LMNA mutation could be regarded as a genetic predisposing factor for "high-risk" advanced AV block. Genetic screening of LMNA might be beneficial for risk stratification and clinical management of patients with advanced AV block. Screening of the LMNA mutations may be useful in middleaged patients (about 45 years old) with advanced AV block, especially those with a familial background.

\section{Limitations of the study}

Several limitations should be mentioned for the present study. First, it was not designed as a regional or populationbased epidemiological study. Thus, the exact prevalence of LMNA mutations in general AV block or DCM populations 
could not be determined from this study. Second, the number of affected family members was not sufficient for clinical data collection. Although, the clinical features of $L M N A$ mutation carriers were considerably unique and specific, we could not exclude bias from the small group of patients. Third, this study could not establish the usefulness of genetic screening of $L M N A$ to the patients studied. Future prospective study is needed to verify the usefulness of $L M N A$ mutation screening.

\section{Conclusion}

We found $L M N A$ mutations in familial cardiomyopathy with insignificant LV remodeling and early-age onset of advanced AV block, followed by ventricular arrhythmia and poor prognosis. Genetic screening of LMNA may be useful for risk stratification and clinical management of middleaged patients with advanced AV block.

\section{Acknowledgments}

We are grateful to the patients and their families who participated in this study. We appreciate the technical assistance received from Ms. Rieko Miura. We are also thankful to the Japanese Ministry of Education, Culture, Sports, Science and Technology for their grant-in-aid (15590716) that supported this study.

\section{References}

Arbustini, E., Pilotto, A., Repetto, A., Grasso, M., Negri, A., Diegoli, M., Campana, C., Scelsi, L., Baldini, E., Gavazzi, A. \& Tavazzi, L. (2002) Autosomal dominant dilated cardiomyopathy with atrioventricular block: a lamin A/C defect-related disease. J. Am. Coll. Cardiol., 39, 981-990.

Benson, D.W. (2004) Genetics of atrioventricular conduction disease in humans. Anat Rec A Discov Mol. Cell Evol. Biol., 280, 934-939.

Brodsky, G.L., Muntoni, F., Miocic, S., Sinagra, G., Sewry, C. \& Mestroni, L. (2000) Lamin A/C gene mutation associated with dilated cardiomyopathy with variable skeletal muscle involvement. Circulation, 101, 473-476.

Broers, J.L., Machiels, B.M., Kuijpers, H.J., Smedts, F., van den Kieboom, R., Raymond, Y. \& Ramaekers, F.C. (1997) A- and B-type lamins are differentially expressed in normal human tissues. Histochem. Cell Biol., 107, 505-517.

Broers, J.L., Ramaekers, F.C., Bonne, G., Yaou, R.B. \& Hutchison, C.J. (2006) Nuclear lamins: laminopathies and their role in premature ageing. Physiol. Rev., 86, 967-1008.

Brunner, M., Olschewski, M., Geibel, A., Bode, C. \& Zehender, M. (2004) Long-term survival after pacemaker implantation. Prognostic importance of gender and baseline patient characteristics. Eur. Heart J., 25, 88-95.

Burke, B. \& Stewart, C.L. (2002) Life at the edge: the nuclear envelope and human disease. Nat. Rev. Mol. Cell Biol., 3, 575-585.

Eriksson, M., Brown, W.T., Gordon, L.B., Glynn, M.W., Singer J., Scott, L., Erdos, M.R., Robbins, C.M., Moses, T.Y., Berglund, P., Dutra, A., Pak, E., Durkin, S., Csoka, A.B., Boehnke, M., Glover, T.W. \& Collins, F.S. (2003) Recurrent de novo point mutations in lamin A cause Hutchinson-Gilford progeria syndrome. Nature, 423, 293-298.

Fatkin, D., MacRae, C., Sasaki, T., Wolff, M.R., Porcu, M., Frenneaux, M., Atherton, J., Vidaillet, H.J. Jr., Spudich, S., De Girolami, U., Seidman, J.G., Seidman, C., Muntoni, F., Muehle, G., Johnson, W. \& McDonough, B. (1999) Missense mutations in the rod domain of the lamin $\mathrm{A} / \mathrm{C}$ gene as causes of dilated cardiomyopathy and conduction-system disease. $N$. Engl. J. Med., 341, 1715-1724.

Hasegawa, A., Sekiguchi, M., Hasumi, M., Take, M., Hosoda, S., Nishikawa, T. \& Hiroe, M. (1990) High incidence of significant pathology in endomyocardial biopsy and familial occurrence in cases with arrhythmia and/or conduction disturbance. Heart Vessels, Supp. 5, 28-30.

Hennekam, R.C. (2006) Hutchinson-Gilford progeria syndrome: review of the phenotype. Am. J. Med. Genet. A, 140, 2603-2624.

Jakobs, P.M., Hanson, E.L., Crispell, K.A., Toy, W., Keegan, H., Schilling, K., Icenogle, T.B., Litt, M. \& Hershberger, R.E. (2001) Novel lamin A/C mutations in two families with dilated cardiomyopathy and conduction system disease. J. Card. Fail., 7, 249-256.

Kass, S., MacRae, C., Graber, H.L., Sparks, E.A., McNamara, D., Boudoulas, H., Basson, C.T., Baker, P.B. 3rd, Cody, R.J., Fishman, M.C., Cox, N., Kong, A., Wooley, C.F., Seidman, J.G. \& Seidman, C.E. (1994) A gene defect that causes conduction system disease and dilated cardiomyopathy maps to chromosome 1p1-1q1. Nat. Genet., 7, 546-551.

Lin, F. \& Worman, H.J. (1993) Structural organization of the human gene encoding nuclear lamin A and nuclear lamin C. $J$. Biol. Chem., 268, 16321-16326.

Maron, B.J., Towbin, J.A., Thiene, G., Antzelevitch, C., Corrado, D., Arnett, D., Moss, A.J., Seidman, C.E. \& Young, J.B. (2006) Contemporary definitions and classification of the cardiomyopathies: an American Heart Association Scientific Statement from the Council on Clinical Cardiology, Heart Failure and Transplantation Committee; Quality of Care and Outcomes Research and Functional Genomics and Translational Biology Interdisciplinary Working Groups; and Council on Epidemiology and Prevention. Circulation, 113, 1807-1816.

Mestroni, L., Maisch, B., McKenna, W.J., Schwartz, K., Charron, P., Rocco, C., Tesson, F., Richter, A., Wilke, A. \& Komajda, M. (1999) Guidelines for the study of familial dilated cardiomyopathies. Collaborative Research Group of the European Human and Capital Mobility Project on Familial Dilated Cardiomyopathy. Eur. Heart J., 20, 93-102.

Meune, C., Van Berlo, J.H., Anselme, F., Bonne, G., Pinto, Y.M. \& Duboc, D. (2006) Primary prevention of sudden death in patients with lamin A/C gene mutations. N. Engl. J. Med., 354, 209-210.

Olson, T.M., Michels, V.V., Ballew, J.D., Reyna, S.P., Karst, M.L., Herron, K.J., Horton, S.C., Rodeheffer, R.J. \& Anderson, J.L. (2005) Sodium channel mutations and susceptibility to heart failure and atrial fibrillation. JAMA, 293, 447-454.

Otomo, J., Kure, S., Shiba, T., Karibe, A., Shinozaki, T., Yagi, T., Naganuma, H., Tezuka, F., Miura, M., Ito, M., Watanabe, J., Matsubara, Y. \& Shirato, K. (2005) Electrophysiological and histopathological characteristics of progressive atrioventricular block accompanied by familial dilated cardiomyopathy caused by a novel mutation of lamin $\mathrm{A} / \mathrm{C}$ gene. J. Cardiovasc. Electrophysiol., 16, 137-145.

Rober, R.A., Weber, K. \& Osborn, M. (1989) Differential timing of nuclear lamin $\mathrm{A} / \mathrm{C}$ expression in the various organs of the mouse embryo and the young animal: a developmental study. Development, 105, 365-378.

Schoeller, R., Andresen, D., Buttner, P., Oezcelik, K., Vey, G. \& Schroder, R. (1993) First- or second-degree atrioventricular block as a risk factor in idiopathic dilated cardiomyopathy. Am. J. Cardiol., 71, 720-726.

Schott, J.J., Alshinawi, C., Kyndt, F., Probst, V., Hoorntje, T.M., Hulsbeek, M., Wilde, A.A., Escande, D., Mannens, M.M. \& Le Marec, H. (1999) Cardiac conduction defects associate with mutations in SCN5A. Nat. Genet., 23, 20-21.

Sekiguch,i M., Hasegawa, A., Hiroe, M., Morimoto, S. \& Nishikawa, T. (2008) Inclusion of electric disturbance type 
cardiomyopathy in the classification of cardiomyopathy: a current review. J. Cardiol., 51, 81-88.

Shimizu, M., Ino, H., Yasuda, T., Fujino, N., Uchiyama, K., Mabuchi, T., Konno, T., Kaneda, T., Fujita, T., Masuta, E., Katoh, M., Funada, A. \& Mabuchi, H. (2005) Gene mutations in adult Japanese patients with dilated cardiomyopathy. Circ. J., 69, 150-153.

Stuurman, N., Heins, S. \& Aebi, U. (1998) Nuclear lamins: their structure, assembly, and interactions. J. Struct. Biol., 122, 42-66.

Taylor, M.R., Fain, P.R., Sinagra, G., Robinson, M.L., Robertson, A.D., Carniel, E., Di Lenarda, A., Bohlmeyer, T.J., Ferguson, D.A., Brodsky, G.L., Boucek, M.M., Lascor, J., Moss, A.C., Li, W.L., Stetler, G.L., Muntoni, F., Bristow, R. \& Mestroni, L. (2003) Natural history of dilated cardiomyopathy due to lamin A/C gene mutations. J. Am. Coll. Cardiol., 41, 771-780.

van Berlo, J.H., de Voogt, W.G., van der Kooi, A.J., van Tintelen, J.P., Bonne, G., Yaou, R.B., Duboc, D., Rossenbacker, T., Heidbuchel, H., de Visser, M., Crijns, H.J. \& Pinto, Y.M. (2005) Meta-analysis of clinical characteristics of 299 carriers of LMNA gene mutations: do lamin $\mathrm{A} / \mathrm{C}$ mutations portend a high risk of sudden death? J. Mol. Med., 83, 79-83.

Waller, B.F., Gering, L.E., Branyas, N.A. \& Slack, J.D. (1993) Anatomy, histology, and pathology of the cardiac conduction system-Part V. Clin. Cardiol., 16, 565-569.

Wolf, C.M., Wang, L., Alcalai, R., Pizard, A., Burgon, P.G., Ahmad, F., Sherwood, M., Branco, D.M., Wakimoto, H., Fishman, G.I., See, V., Stewart, C.L., Conner, D.A., Berul, C.I., Seidman, C.E. \& Seidman, J.G. (2008) Lamin A/C haploinsufficiency causes dilated cardiomyopathy and apoptosis-triggered cardiac conduction system disease. Mol. Cell Cardiol., 44, 293-303.

World Health Organization / International Society and Federation of Cardiology Task Force. (1996) Report of the 1995 World Health Organization / International Society and Federation of Cardiology Task Force on the Definition and Classification of Cardiomyopathies. Circulation, 93, 841-842.

Xiao, H.B., Roy, C., Fujimoto, S. \& Gibsonm, D.G.. (1996) Natural history of abnormal conduction and its relation to prognosis in patients with dilated cardiomyopathy. Int. J. Cardiol., 53, 163-170. 